\title{
Computations of Ground State and Excitation Energies of Poly(3-methoxy-thiophene) and Poly(thienylene vinylene) from First Principles
}

\author{
A.V. Gavrilenko, S.M. Black, A.C. Sykes, C.E. Bonner, and V.I. Gavrilenko \\ Center for Materials Research, Norfolk State University \\ 700 Park Ave, Norfolk, VA 23504 \\ a.v.gavrilenko@nsu.edu
}

\begin{abstract}
Ground state and excitation energies of poly(3-methoxy-thiophene) (PMT) and poly(thienylene vinylene) (PTV) conjugated polymers are studied by first principles density functional theory (DFT). Two basic approaches of computational chemistry and physics are compared: time dependent DFT (TDDFT) of clusters and $a b$ initio pseudopotentials within a standard DFT (PP-DFT) of infinite polymer chains. We demonstrate that series of excitation energies of PMT calculated by TDDFT with increased unit numbers converge well to the real experimentally measured energy gaps. Combination of TDDFT cluster method with PPDFT approach for infinite chain provides single-gap quasiparticle correction value needed for optical calculations. Infinite chain model is used to calculate optical absorption of PTV.
\end{abstract}

Keywords: Density functional theory, equilibrium geometry, excitation energies, conjugated polymers.

\section{Introduction}

The search for inexpensive renewable energy sources has sparked considerable interest in the development of photovoltaics based on conjugated polymers and organic molecules [12]. In organic materials, the dominant light absorption interesting for applications is caused by $\pi$-electron transitions between highest occupied molecular orbital (HOMO) and excited lowest unoccupied molecular orbital (LUMO) 34. The spectral location of the optical absorption band (or HOMO-LUMO gap value) determines efficiency of photovoltaic and optoelectronic devices 31516 . It has been understood earlier that the gap value in poly $(3-$ methoxy-thiophene) (PMT) and poly(thienylene vinylene) (PTV) is determined by the rings and less affected by the sidegroups 7 .

The technologically challenging goal of bandgap engineering could be substantially supported by modeling and simulation in addition to highlighting the methods of materials modification to achieve many of the desired properties. However, realistic modeling of the ground state and excitation energy of conjugated polymers, which are required for optics, still remains a challenging task for 
first principle theories 8910]. In the present work we compare different computational approaches for realistic predictions of the ground and excited states of conjugated polymers. The first approach involves modeling of atomic structure of conjugated polymers as clusters [711. The excitation energies are calculated by Time Dependent Density Functional Theory (TDDFT). The second approach involves modeling of conjugated polymers as infinitely long chains thus allowing application of $a b$ initio pseudopotentials within DFT (PP-DFT). We also present calculated optical absorption spectra and discuss both computational approaches in comparison to each other and to the data available in literature.

\section{Methods}

Density functional theory (DFT) has been shown for decades to be very successful for the ground state analysis of different materials 12 . In order to be able to predict physical properties of conjugated polymer systems it is important to realistically describe both short (covalent) and long range (Coulomb and van der Waals, vdW) components of intermolecular interactions 8]. Local density (LDA) 13 and generalized gradient approximations (GGA) 14 are frequently used to account for the exchange and correlation (XC) interaction. Coulomb part dominates intermolecular potential energy over higher order vdW part. The Coulomb interaction is realistically reproduced by LDA, but the vdW interactions is not included in standard DFT. It has been shown that equilibrium distance between organic molecule and solid (graphene) surface predicted by LDA is in good agreement to the value followed from explicit inclusion of the vdW into the interaction Hamiltonian [8] thus justifying neglect of the vdW interaction for interatomic equilibrium distances predictions.

In this work the TDDFT method as implemented in the Gaussian03 package 1514 was used for cluster calculations of PMT. For atomic structure optimization the B3LYP/6-31G* basis was used. Excitation energies were obtained through extrapolation of the values calculated for $n=2$ to 7 repeat units. The calculated data for the $n=1$ case does not obey the $1 / n$-extrapolation due to less pronounced delocalization of $\pi$-electrons in a single unit, and relevant data isn't considered here. Similar approach to obtain ground and excited electronic states of PMT infinite chain by extrapolation of TDDFT cluster data was used in 11. According to our finding the differences in excitation energies if using B3LYP/6-31G* and B3LYP/6-31+G* basis sets become negligible for $n>5$ as shown in Table 1. Effect of the basis could be an interesting point for future studies in the field, however, it is out of scope of this work. Solvent effects were not taken into account for the cluster calculations.

In addition, the PMT and PTV conjugated polymers were also modeled as infinite atomic chains 910 in two parts. We used the $\mathrm{DMol}^{3}$ [1617 and CASTEP 18. (based on ab initio pseudopotentials, PP) computational packages as implemented in Materials Studio. First, the geometry relaxation was performed using the DFT-LDA method as implemented in $\mathrm{DMol}^{3}$. Equilibrium atomic structures were obtained by LDA method. Only Coulombic interaction providing most 
Table 1. Basis set effect on the HOMO-LUMO gap and excitation energies of 3methoxy-thiophene in $\mathrm{eV}$

\begin{tabular}{ccccc} 
& \multicolumn{2}{c}{ HOMO-LUMO Gap } & \multicolumn{2}{c}{ Excitation Energies } \\
Oligomer Units & $6-31 \mathrm{G}^{*}$ & $6-31+\mathrm{G}^{*}$ & $6-31 \mathrm{G}^{*}$ & $6-31+\mathrm{G}^{*}$ \\
\hline 2 & 3.97 & 3.92 & 3.76 & 3.66 \\
3 & 3.19 & 3.16 & 3.01 & 2.94 \\
4 & 2.65 & 2.62 & 2.49 & 2.43 \\
5 & 2.36 & 2.33 & 2.19 & 2.14 \\
6 & 2.16 & $\mathrm{n} / \mathrm{a}$ & 1.98 & $\mathrm{n} / \mathrm{a}$ \\
7 & 2.02 & $\mathrm{n} / \mathrm{a}$ & 1.83 & $\mathrm{n} / \mathrm{a}$ \\
\hline
\end{tabular}

important contribution to the interchain interaction [8] is included. The unit cell is replicated in all 3 dimensions, the height, $d 3$, was set to be $15 \AA$ in order to quench the interaction between the thiophene ring and the methyl group. We obtained very weak effect of the $d 3$ on optics therefore this value was constrained.

The fully relaxed structure was then used to calculate the electronic and optical properties of the conjugated polymer within PP-DFT as implemented in CASTEP. Three dimensional periodicity in this case allows application of $a b$ initio pseudopotential method which makes optical calculations straight forward [19. Optical absorption spectra of the PTV conjugated polymers are calculated within the independent particles picture (random phase approximation, RPA) employing ultrasoft pseudopotentials [9]. Details of the optical calculations are described in the detail in Ref. [20].

\section{Results and Discussion}

We studied equilibrium atomic configurations and excitation energies of PMT and PTV using different computational approaches described above. First we consider results of cluster calculations of PMT. As stated above the excitation energies are obtained by extrapolation of the values predicted for 2 to 7 units to the infinitely long chain.

Our previous work with substituted poly(phenylene vinylenes) (PPV) has resulted in the observation of supramolecular scale quasicrystalline arrangements of these polymers with distinctive unit cell dimensions [192122]. In those reports, both experimental and computational methods were used to confirm the formation of crystalline domains of the PPV conjugated polymer with interchain dimensions on the order of the van der Waals radii which would be expected to lead to a strong interchain interaction.

In the present work, we extend these methods to the study of two more technologically relevant conjugated polymers, poly(3-methoxy-thiophene) and poly(thienylene vinylene). These conjugated polymers are similar to PPV but contain five membered rings with sulfur substitution in the ring and as a result a lower bandgap than their PPV counterparts. Each polymer has an alkoxy substituent on the thiophene ring which donates electrons to the chain leaving 


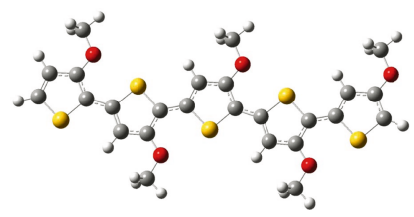

(a) PMT Cluster

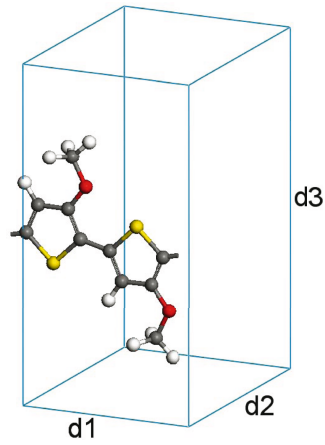

(b) PMT unit cell

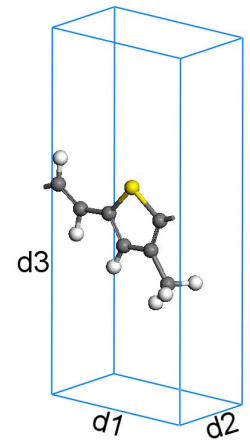

(c) PTV unit cell

Fig. 1. (a) Converged atomic structure of pentamer (5 units) of 3-methoxy-thiophene. $3 \mathrm{D}$-view of the poly(3-methoxy-thiophene) (b) and poly(thienylene vinylene) (c) unit cells. Optimized unit cell dimensions for (b) are $d 1=7.74 \AA$, $d 2=10 \AA$, and $d 3=15$ $\AA$, and for (c) are $d 1=6.55 \AA, d 2=3.75 \AA$, and $d 3=15 \AA$.

the chain slightly electron rich forming an $n$-type material. Experimentally, an eight carbon (or longer) alkoxy chain is used in part to assist the solvation properties of the polymer in organic solvents. In this work, the alkoxy side chain was shortened to a single carbon methoxy chain as the chain length does not affect the electron donating ability of the alkoxy subtstituent and the arrangement of the chains did not include solvent effects. Additionally, the shorter methoxy side chain allows the computational resources to be reserved for the conjugated backbone.

\subsection{Equilibrium Geometry}

Fully relaxed configuration of 3-methoxy-thiophene pentamer is shown in Fig 1(a) as an example. Geometries of PMT and PTV polymers modeled as an infinite chain are given in Figs. 1(b) and 1(c). Interchain interaction is an important aspect since the close proximity of neighboring chains will split the electronic states of the polymer, thereby reducing the bandgap and creating additional states. As mentioned above the $d 3$ provides very weak effect on optics therefore this value was constrained to $15 \AA$. The equilibrium intermolecular distances (d1) for PMT and PTV are $7.74 \AA$ and $6.55 \AA$, respectively, and were determined by cluster calculations of a single polymer chain.

\subsection{Excitation Energies}

The excitation energies (EXC) were calculated using both the TDDFT theory and $a b$ initio pseudopotential method as described in section 2 In order to obtain correct values for EXC in PTV within the TDDFT we studied convergence of the EXC with increase of the unit number in the polymer chain. Predicted EXC values are given in Table 1 In Fig. 2 we demonstrate that calculated EXC data 


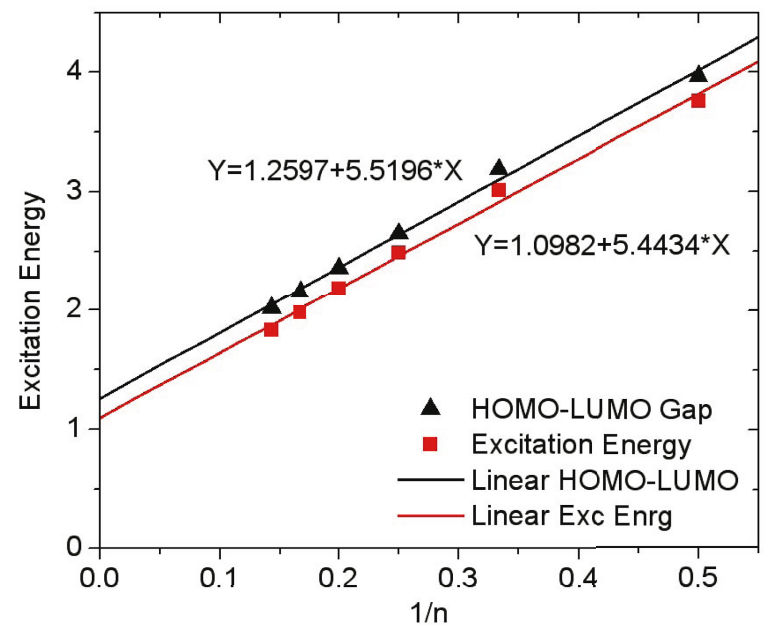

Fig. 2. Energy extrapolation to infinite chain length for both the HOMO-LUMO gap and the excitation energy. The excitation energy yields a value of $1.1 \mathrm{eV}$ which is lower than that of the HOMO-LUMO gap by about $0.1 \mathrm{eV}$.

could be fitted by $1 / n$ function, where $n$ is the number of units in the chain. The observed $1 / n$ dependence allows simple extrapolation to infinitely long polymer chain as shown in Fig. 2, however, analysis of this dependence requires further study.

The obtained TDDFT excitation energy value of $1.1 \mathrm{eV}$ is compared with that calculated by PP-DFT method. The excitation energies of PMT polymers are obtained from the Projected Density Of States (PDOS). The PDOS spectrum of PMT infinite chain polymer is given in Fig. 3.

The calculated PP-DFT HOMO-LUMO gap of $0.9 \mathrm{eV}$ (excitation energy) is lower then TDDFT value by $0.2 \mathrm{eV}$. It is well known that DFT excitation energies underestimate the actual energy gaps. In order to obtain realistic values the quasiparticle (QP) correction to the DFT data, substantial complications of computations are required 23 . On the other hand the QP values for the HOMOLUMO gap could be obtained from the comparison with available experimental data or with TDDFT gap-values 23. By comparison with optical absorption data we demonstrate that both methods provide very close results.

\subsection{Optical Absorption}

Calculated optical absorption spectrum of PTV conjugated polymers is shown in comparison with experimental data 2419 in Fig. 4. In order to match spectral location of theoretical spectrum to the experimental one (to the dominant peak located at $577 \mathrm{~nm}$ ) a QP correction (blue shift) of $0.18 \mathrm{eV}$ was applied to the calculated data in Fig. 4. The QP corrected theoretical spectrum correctly reproduces shoulders on a long-frequency wing of the spectrum. These shoulders are attributed to molecular vibrations (at $619 \mathrm{~nm}$ ) and to the aggregation (at 


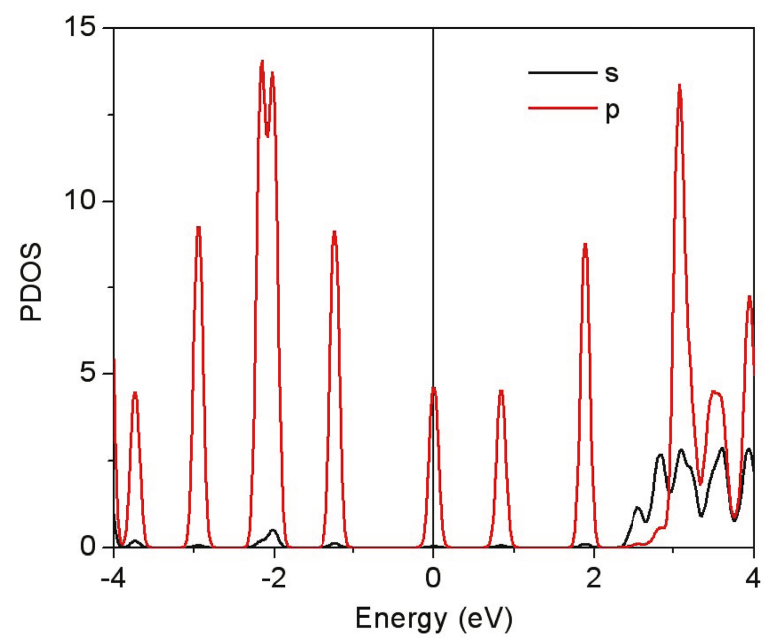

Fig. 3. Electronic structure of 3-methoxy-thiophene in the form of Projected Density of States (PDOS)

$685 \mathrm{~nm}$ ) caused by interchain interaction [19. The shape of the calculated spectrum, however, shows remarkable differences from experimental data (see Fig. 4). It has been demonstrated before that for optical line shape analysis inclusion of excitonic effects in polymers is necessary [10. However this technically challenging theory of optical response in polymers is out of scope of present study. Inclusion of exciton effects is expected to improve comparison between the line shapes of predicted and measured optical absorption spectra which should be addressed in future works.

From the comparison between calculated and measured optical absorption spectra of PTV we obtained the QP correction value of $0.18 \mathrm{eV}$ (the blue-shift) which is applied to the calculated spectrum in order to match the experimental data. The QP value of $0.18 \mathrm{eV}$ obtained for PTV polymers is very close to the DFT gap underestimation (by $0.2 \mathrm{eV}$ ) which we obtained for PMT polymers from comparison to our TDDFT value. The TDDFT data realistically predict experimental HOMO-LUMO gap values in conjugated polymers 11. The DFT+QP calculated gap values agree well with those predicted by TDDFT method for both organic and inorganic materials [23. This comparison suggests that excitation energies of the PMT polymer obtained by extrapolation of TDDFT values to an infinitely long chain correctly reproduces a real HOMO-LUMO gap in agreement with general conclusions followed from comparative DFT+QP and TDDFT analysis [1123].

Therefore, results of this work demonstrate that relatively simple TDDFT cluster calculations combined with extrapolation method provide correct QP values for HOMO-LUMO gap which could be used with PP-DFT method for realistic predictions of optical absorption in conjugated polymers. This is substantial simplification of the theory which still incorporates important many-body 


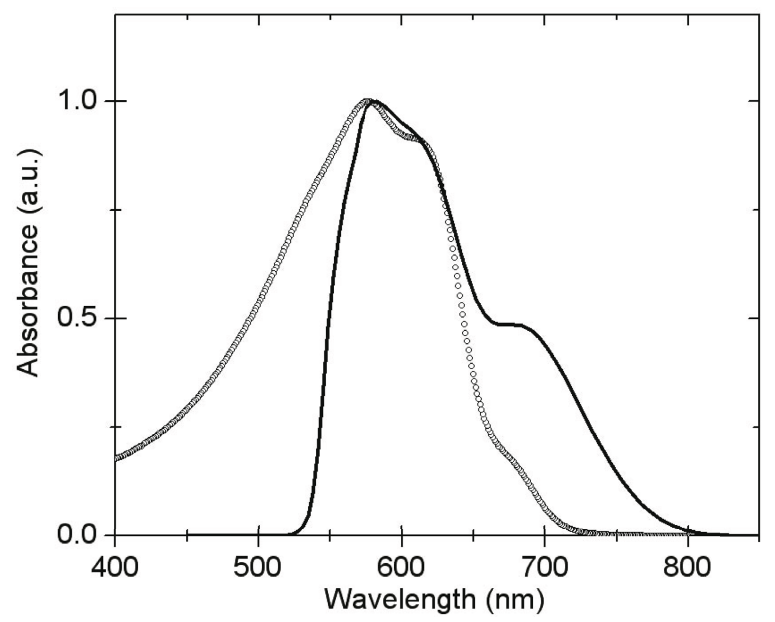

Fig. 4. Calculated optical absorption spectra resulted PTV (solid) in comparison with experimental data (circles) measured in 24]

effects in polymer optics. Thus for conjugated polymers, the realistic first principle predictions of optical spectra could be performed within a simplified scheme avoiding large-scale computations.

\section{Conclusions}

In this work we present results of the ground state and excitation energies study of poly(3-methoxy-thiophene) (PMT) and poly(thienylene vinylene) (PTV) conjugated polymers by first principles density functional theory (DFT). We used two approaches for computations of excitation states: time dependent DFT (TDDFT) of clusters and ab initio pseudopotentials based DFT (PP-DFT) of infinite polymer chains. The excitation energies of PMT calculated by TDDFT with increased unit numbers converge well to the real experimentally measured energy gaps. Combination of TDDFT cluster method with PP-DFT approach for infinite chain provide single-gap quasiparticle corrections value needed for optical calculations. Infinite chain model is used to calculate optical absorption of PTV. Predicted optical absorption spectrum is in good agreement with experiment. Combination of both cluster TDDFT and infinite chain PP-DFT methods is rather simple and promising first principle approach for realistic modeling and simulations of conjugated polymers optics.

Acknowledgments. This work is supported by NSF STC DMR-0120967, NSF PREM DRM-0611430, NSF NCN EEC-0228390, and NASA CREAM NCC31035. 


\section{References}

1. Dhanabalan, A., van Duren, J.K.J., van Hal, P.A., van Dongen, J.L.J., Janssen, R.A.J.: Synthesis and characterization of a low bandgap conjugated polymer for bulk heterojunction photovoltaic cells. Adv. Funct. Mater. 11, 255-262 (2001)

2. Sun, S.S., Sariciftci, N.S.: Organic Photovoltaics: Mechanisms, Materials, and Devices. CRC Press, Boca Raton (2005)

3. Patil, A.O., Heeger, A.J., Wudl, F.: Optical properties of conducting polymers. Chem. Rev. 88, 183-200 (1988)

4. Skotheim, T.A. (ed.): Handbook of Conducting Polymers, 2nd edn. CRC Press, Boca Raton (1997)

5. Eckhardt, H., Shacklette, L.W., Jen, K.Y., Elsenbaumer, R.L.: The electronic and electrochemical properties of poly(phenylene vinylenes) and poly(thienylene vinylenes): An experimental and theoretical study. J. Chem. Phys. 91, 1303-1315 (1989)

6. Blohm, M.L., Pickett, J.E., Van Dort, P.C.: Synthesis, characterization, and stability of poly(3,4-dibutoxythiophenevinylene) copolymers. Macromol. 26, 2704-2710 (1993)

7. Toussaint, J.M., Bredas, J.L.: Theoretical analysis of the geometric and electronic structure of small-band-gap polythiophenes: poly(5,5'-bithiophene methine) and its derivatives. Macromol. 26, 5240-5248 (1993)

8. Ortmann, F., Schmidt, W.G., Bechstedt, F.: Attracted by long-range electron correlation: Adenine on graphite. Phys. Rev. Lett. 95, 186101-186105 (2005)

9. Gavrilenko, V.I.: Ab initio modeling of optical properties of organic molecules and molecular complexes. In: Alexandrov, V.N., van Albada, G.D., Sloot, P.M.A., Dongarra, J. (eds.) ICCS 2006. LNCS, vol. 3993, pp. 89-96. Springer, Heidelberg (2006)

10. Rohlfing, M., Tiago, M.L., Louie, S.G.: First-principles calculation of optical absorption spectra in conjugated polymers: role of electron-hole interaction. Synth. Met. 116, 101 (2001)

11. Ma, J., Li, S., Jiang, Y.: A time-dependent DFT study on band gaps and effective conjugation lengths of polyacetylene, polyphenylene, polypentafulvene, polycyclopentadiene, polypyrrole, polyfuran, polysilole, polyphosphole, and polythiophene. Macromol. 35, 1109-1115 (2002)

12. Kohn, W., Becke, A.D., Parr, R.G.: Density functional theory of electronic structure. J. Phys. Chem. 100, 12974-12980 (1996)

13. Perdew, J.P., Wang, Y.: Accurate and simple analytic representation of the electron-gas correlation energy. Phys. Rev. B 45, 13244-13249 (1992)

14. Perdew, J.P., Burke, K., Ernzerhof, M.: Generalized gradient approximation made simple. Phys. Rev. Lett. 77, 3865-3868 (1996)

15. Miller, W.H., Hernandez, R., Handy, N.C., Jayatilaka, D., Willets, A.: Ab initio calculation of anharmonic constants for a transition state, with application to semiclassical transition state tunneling probabilities. Chem. Phys. Lett. 172, 62 (1990)

16. Delley, B.: An all-electron numerical method for solving the local density functional for polyatomic molecules. J. Chem. Phys. 92, 508-517 (1990)

17. Delley, B.: From molecules to solids with the $\mathrm{DMol}^{3}$ approach. J. Chem. Phys. 113, 7756-7764 (2000)

18. Segall, M.D., Lindan, P.J.D., Probert, M.J., Pickard, C.J., Hasnip, P.J., Clark, S.J., Payne, M.C.: First-principles simulation: ideas, illustrations and the CASTEP code. J. Phys.Cond. Matt. 14, 2717-2744 (2002) 
19. Gavrilenko, A.V., Matos, T., Bonner, C.E., Sun, S.S., Zhang, C., Gavrilenko, V.I.: Optical absorption of poly(thiophene vinylene) conjugated polymers. Experiment and first principle theory. J. Phys. Chem. B (in press) (2008)

20. Gavrilenko, V.I., Bechstedt, F.: Optical functions of semiconductors beyond density-functional theory and random-phase approximation. Phys. Rev. B 55, 4343-4352 (1997)

21. Bonner, C.E., Charter, S., Lorts, A., Adebolu, O.I., Zhang, C., Sun, S.S., Gavrilenko, V.I.: Luminescence and optical absorption of conjugated polyphenylene-vinylene polymers. In: Proc. of SPIE, vol. 6320, 63200J (2006)

22. Seo, K., Choi, S., Zhang, C., Sun, S.S., Bonner, C.: Processing and molecular packing of a derivatized PPV -donor-bridge-acceptor-bridge- type block copolymer for potential photovoltaic applications. Adv. Mater (in press) (2008)

23. Onida, G., Reining, L., Rubio, A.: Electronic excitations: density-functional versus many-body Green's-function approaches. Rev. Mod. Phys. 74, 601-633 (2002)

24. Matos, T.D.: Design, synthesis, and characterization of 3-dodedyl-2,5-poly(thienylene vinylene) polymer for optoelectronics and solar energy conversions. Master's thesis, Norfolk State University (2007) 